\title{
Graphitic Carbon Nitride Quantum Dots Embedded in Carbon Nanosheets for Near-Infrared Imaging-Guided Combined Photo-Chemotherapy
}

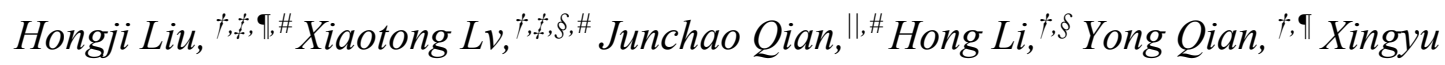
Wang, ${ }^{\dagger, t, \uparrow}$ Xiangfu Meng, ${ }^{\dagger, t,}$ Wenchu Lin, ${ }^{\dagger, \xi}, *$ Hui Wang, ${ }^{\dagger,},, \xi, *$

${ }^{\dagger}$ High Magnetic Field Laboratory, Hefei Institutes of Physical Science, Chinese Academy of Sciences, Hefei 230031, Anhui, P. R. China

$\$$ University of Science and Technology of China, Hefei, 230026, Anhui, P. R. China

II Key Laboratory of Structure and Functional Regulation of Hybrid Materials (Anhui University), Ministry of Education, Hefei, 230601, Anhui, P. R. China

$\S$ Key Laboratory of High Magnetic Field and Ion Beam Physical Biology, Hefei Institutes of Physical Science, Chinese Academy of Sciences, Hefei 230031, Anhui, P.

R. China

" Hefei Cancer Hospital, Anhui Province Key Laboratory of Medical Physics and Technology, Institute of Health and Medical Technology, Hefei Institutes of Physical Science, Chinese Academy of Sciences, Hefei 230031, P. R. China

*E-mail: wenchu@hmfl.ac.cn; hw39@hmfl.ac.cn 
Calculation of quantum yield of CNQD-CN: The relative PL QY of contrast agent is the usually the ratio of the emitted photons to the absorbed photons. ${ }^{1}$ It can be led to $Q Y=Q Y_{\text {ref }} \frac{\eta^{2}}{\eta_{\text {ref }}^{2}} \frac{I}{I_{\text {ref }}} \frac{A_{\text {ref }}}{A}$ where $\mathrm{QY}$ ref is the QY of rhodamine B dissolved in ethanol as a reference, $\eta$ is the refractive index of $\mathrm{H}_{2} \mathrm{O}=1.3333$ ( $\eta_{\text {ref }}$ of ethanol $=1.361$ ), $\mathrm{I}$ is the integrated PL intensity and $\mathrm{A}$ is the absorbance at the excitation wavelength. The absolute PL QY was measured and estimated.

Calculation of photothermal conversion efficiency of CNQD-CN: The photothermal conversion efficiency was calculated based on a reported method, ${ }^{2}$ as follows:

$\eta=\frac{h A \Delta T_{\max }-Q_{s}}{I\left(1-10^{-A} \lambda\right)}$

where $\mathrm{h}$ is the heat transfer coefficient, $\mathrm{A}$ is the surface area of the container that holds CNQD-CN solution. $\triangle \mathrm{T}_{\max }$ is the temperature change $\left(26.8^{\circ} \mathrm{C}\right)$ of $\mathrm{CNQD}-\mathrm{CN}$ solution at the maximum steady-state temperature, I is the laser power (1000 $\left.\mathrm{mW} / \mathrm{cm}^{2}\right), \mathrm{A}_{\lambda}$ is the absorbance of CNQD-CN at $808 \mathrm{~nm}(0.0484), \mathrm{Q}_{\mathrm{s}}$ is the heat associated with the light absorbance of the solvent $(9.0 \mathrm{~mW})$, and $\eta$ is the photothermal conversion efficiency.

$\mathrm{h}_{\mathrm{A}}$ was calculated as follows:

$\mathrm{h}_{\mathrm{A}}=\frac{\mathrm{mC}_{\mathrm{P}}}{\tau_{\mathrm{s}}}(2)$ 
where $\mathrm{m}$ is the mass of CNQD-CN $(0.2 \mathrm{mg}), \mathrm{C}_{\mathrm{P}}$ is the specific heat of carbon $(0.71$ $\mathrm{J} / \mathrm{g}{ }^{\circ} \mathrm{C}$ ), and the system time constant $\tau_{\mathrm{s}}$ is calculated as follows:

$\mathrm{t}=-\tau_{\mathrm{s}} \ln \theta$

Where the $t$ is the cooling time after NIR irradiation was turned off. The $\theta$ is the ratio between $\Delta \mathrm{T}_{\max }$ and $\Delta \mathrm{T}_{\mathrm{t}} . \Delta \mathrm{T}_{\mathrm{t}}$ is temperature change of CNQD-CN solution when the cooling time is t compared with the room temperature of CNQD-CN solution. From Figure $6 \mathrm{c}$, the system time constant $\tau_{\mathrm{s}}$ was evaluated to be $120.81 \mathrm{~s}$.

Thus, from Equation (2), $\mathrm{h}_{\mathrm{A}}$ is calculated to be $1.175 \mathrm{~mW} /{ }^{\circ} \mathrm{C}$. Substituting $\mathrm{h}_{\mathrm{A}}=1.175$ $\mathrm{mW} /{ }^{\circ} \mathrm{C}$ in Equation (1), the photothermal conversion efficiency $\eta$ of CNQD-CN is calculated to be $21.32 \%$.

Calculation of therapeutic efficacies of CNQD-CN: The therapeutic efficacies was calculated based on a reported method. ${ }^{3}$ The additive therapeutic efficacies of independent chemo and phototherapy treatments were estimated using the relation $\mathrm{T}$ additive $=100-\left(\mathrm{f}_{\text {chemo }} \times \mathrm{f}_{\text {phototherapy }}\right) \times 100$, where $\mathrm{f}$ is the fraction of surviving cells after each treatment. The t-test was used to compare the therapeutic efficacies of combined treatment with the additive efficacies of chemo and phototherapy alone. 


\section{Supplementary figures and text}

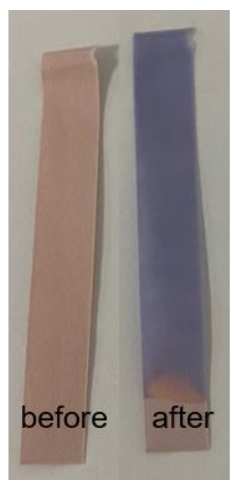

Figure S1. The optical pictures of wet litmus paper before and after contact with the gas from the reactor. The appearance of blue color indicates the production of ammonia gas during the formation of CNQD-CN.

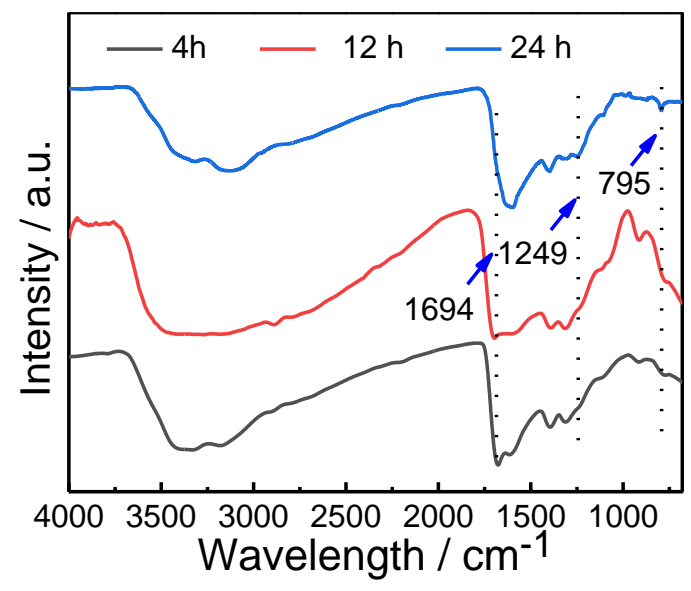

Figure S2. Typical FT-IR spectra of the formed products from formamide decomposition under different reaction time.
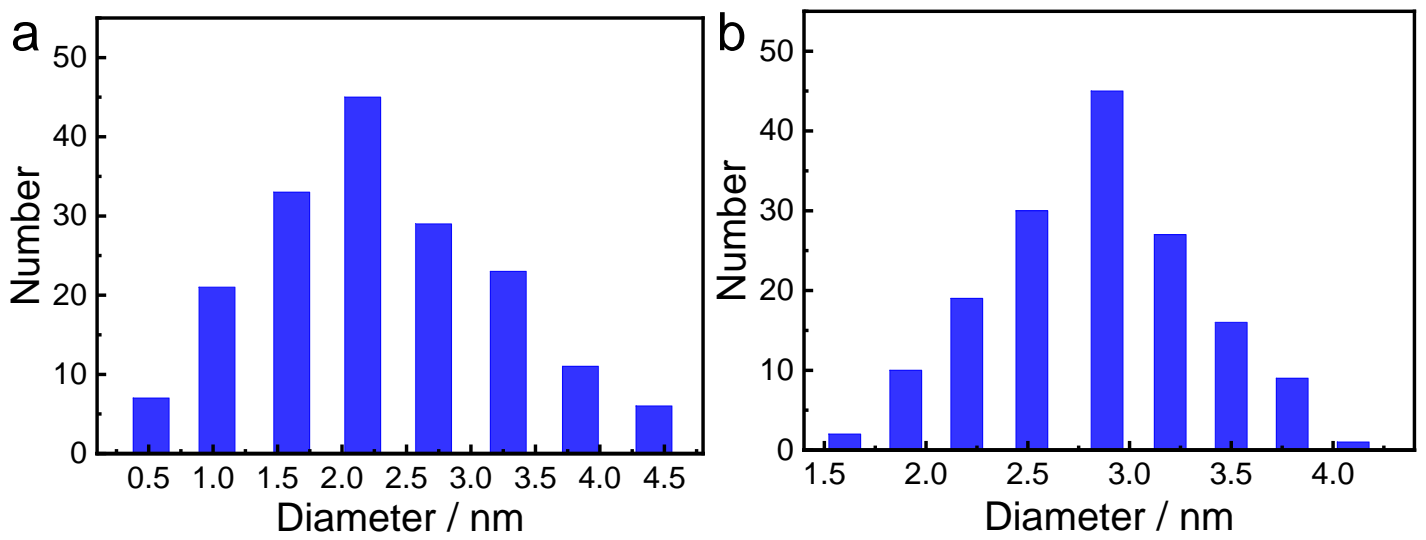


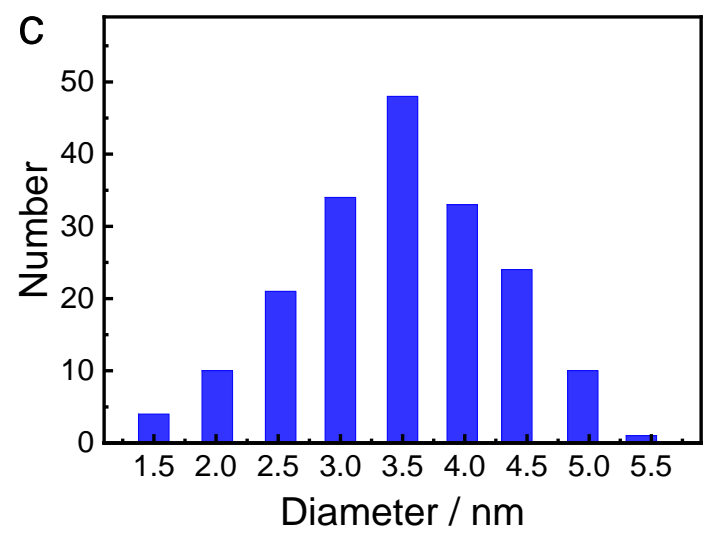

Figure S3. The size distributions of CNQDs synthesized under different reaction temperature (a: $\left.140{ }^{\circ} \mathrm{C} ; \mathrm{b}: 160{ }^{\circ} \mathrm{C} ; 180^{\circ} \mathrm{C}\right)$.

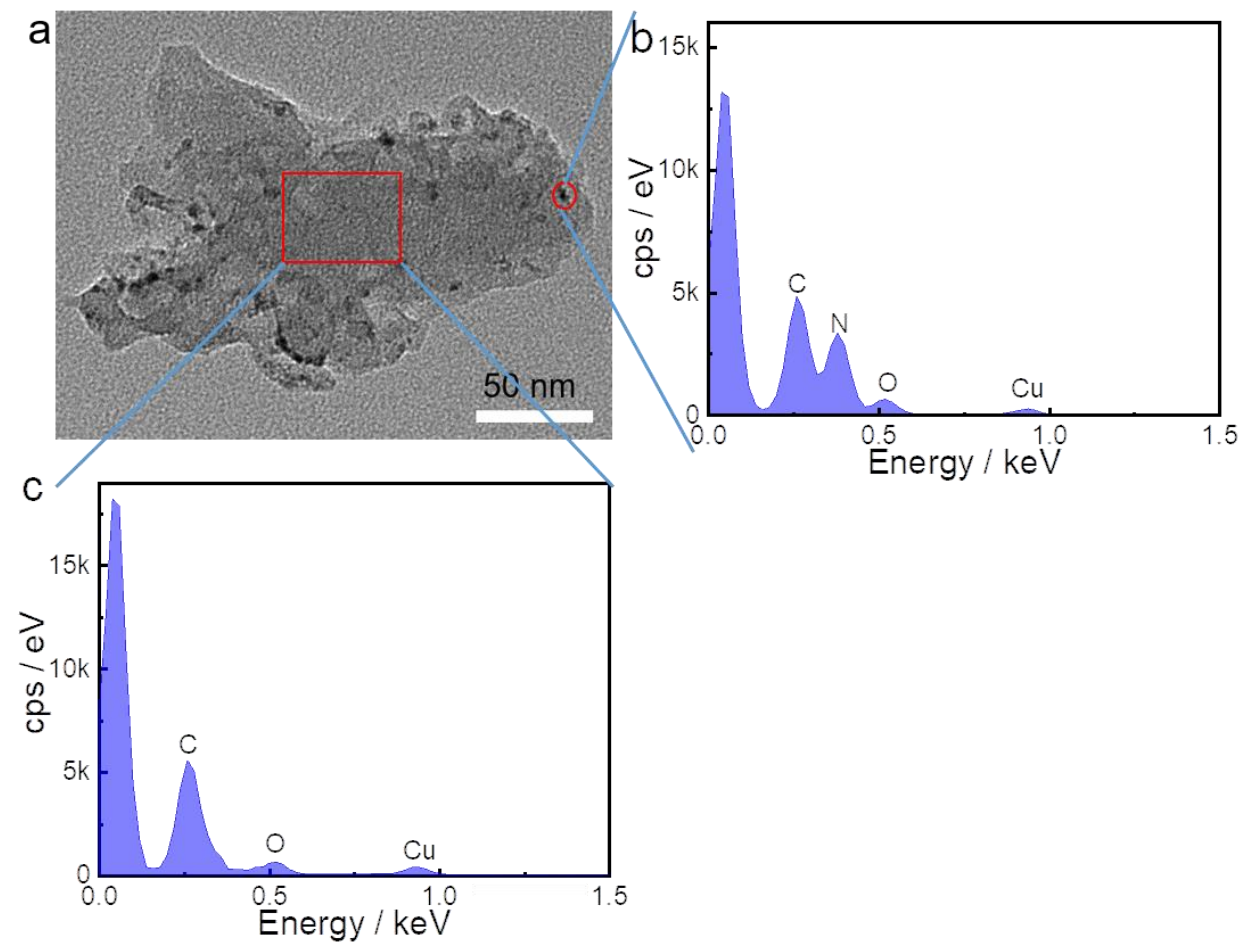

Figure S4. (a) TEM image of CNQD-CN. (b) EDX of single CNQDs embedded in the carbon sheet. (c) EDX of sectional carbon nanosheets from CNQD-CN. 

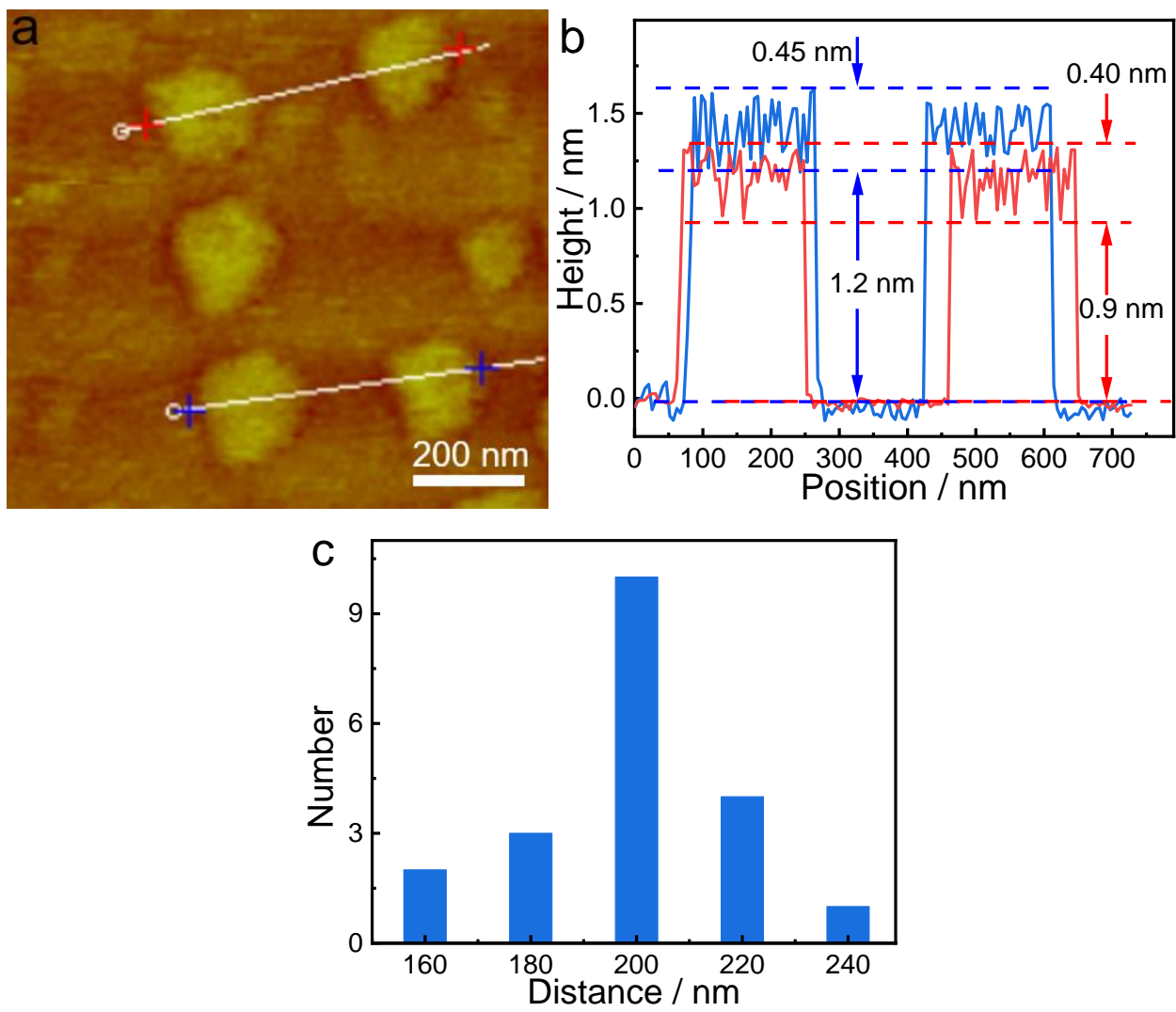

Figure S5. (a) AFM image of CNQD-CN and (b) the corresponding height curve. (c) The size distribution of CNQD-CN.

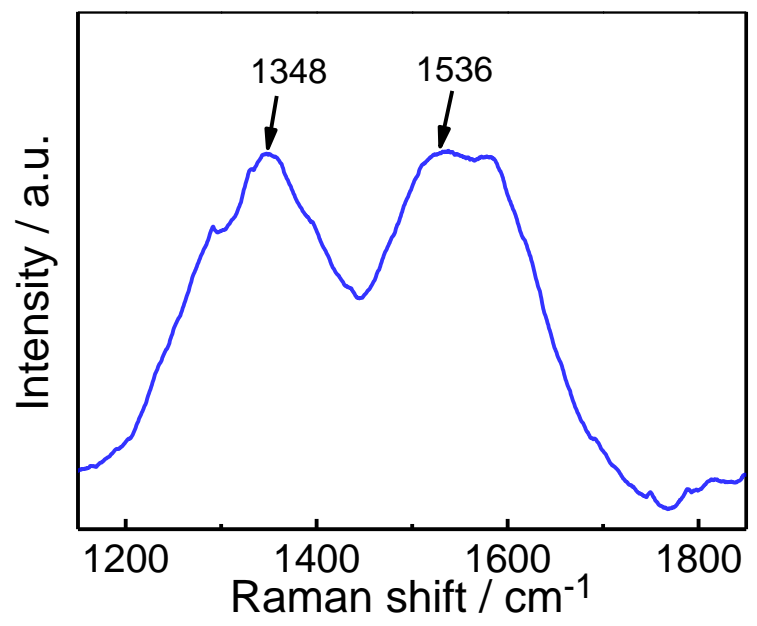

Figure S6. Typical Raman spectrum of the CNQD-CN. 


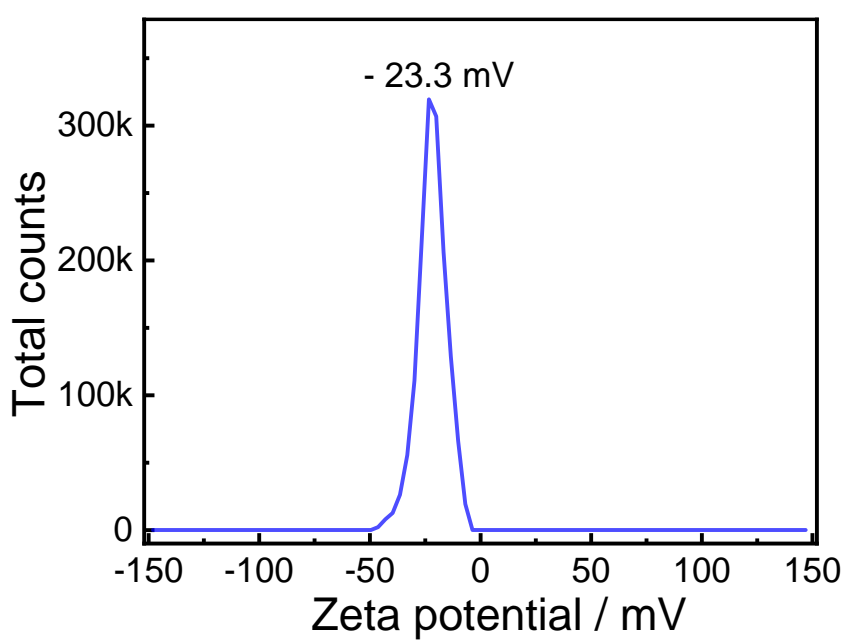

Figure S7. The zeta potential distribution of the CNQD-CN.

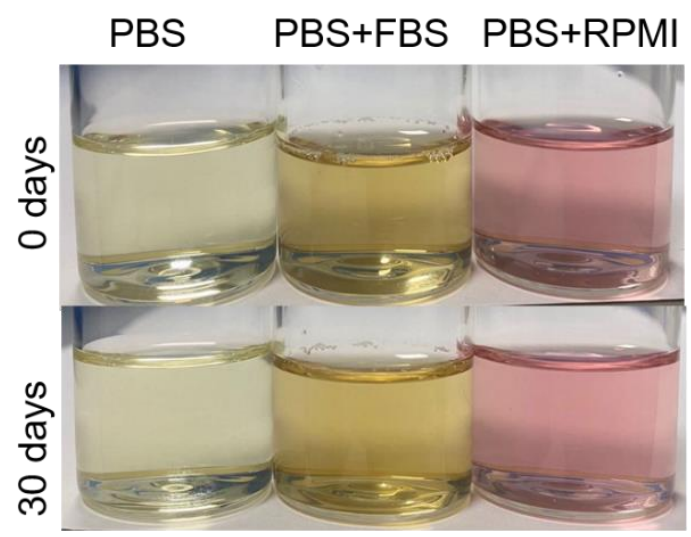

Figure S8. Photographs of CNQD-CN dispersed in different physiological environments after being stored for 0 day (top) and 30 day (bottom).

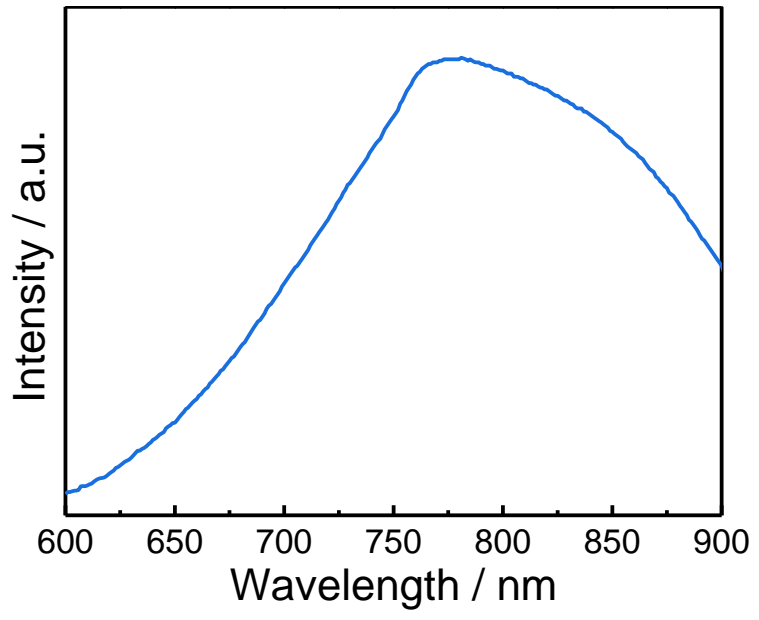

Figure S9. NIR absorption spectra for the CNQD-CN. 

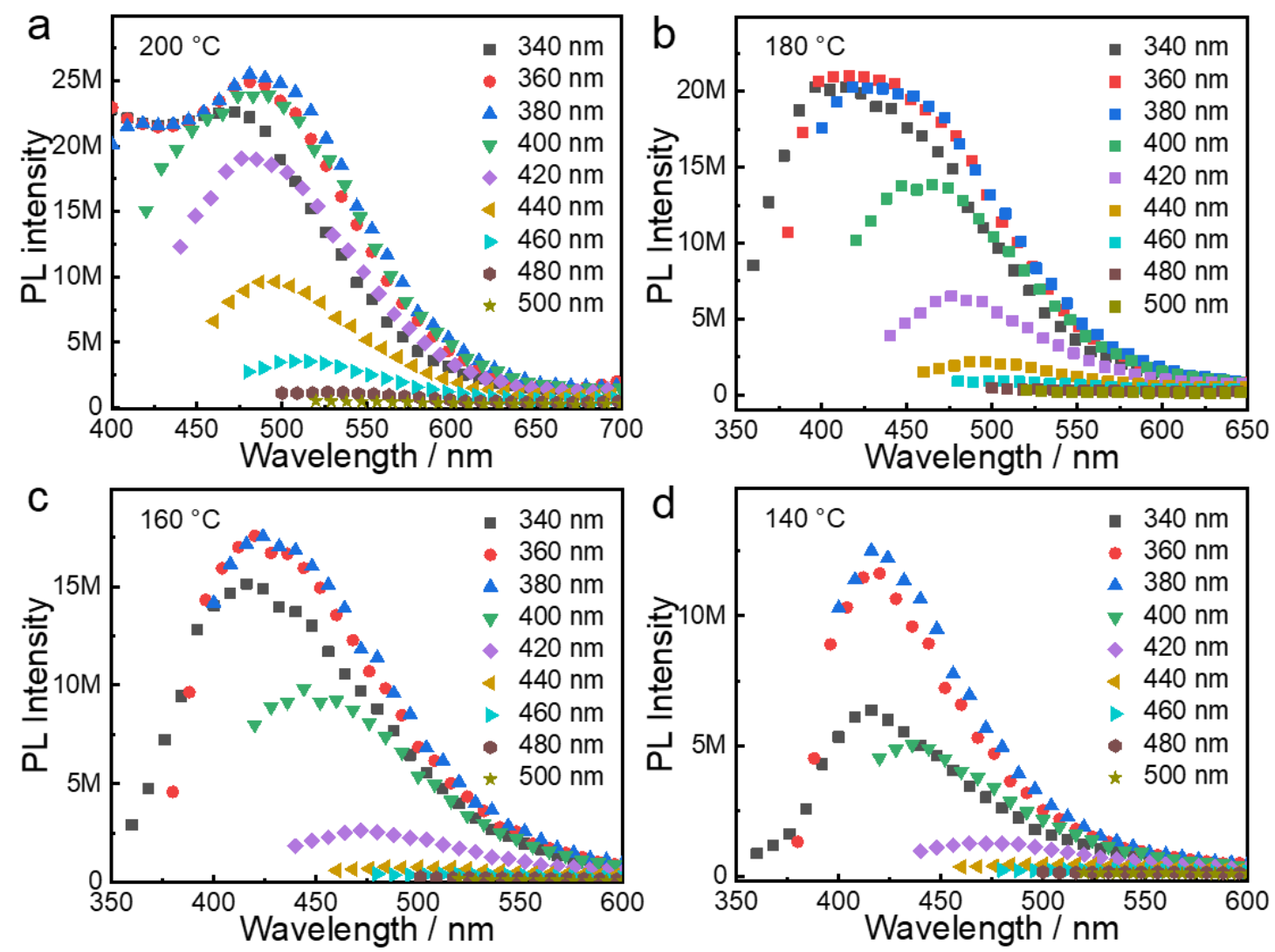

Figure S10. Excitation wavelength-tuned fluorescence spectra of the CNQD-CN synthesized at different reaction temperature. a: $200{ }^{\circ} \mathrm{C}$; b: $180{ }^{\circ} \mathrm{C} ; \mathrm{c}: 160{ }^{\circ} \mathrm{C}$; d: 140 ${ }^{\circ} \mathrm{C}$.

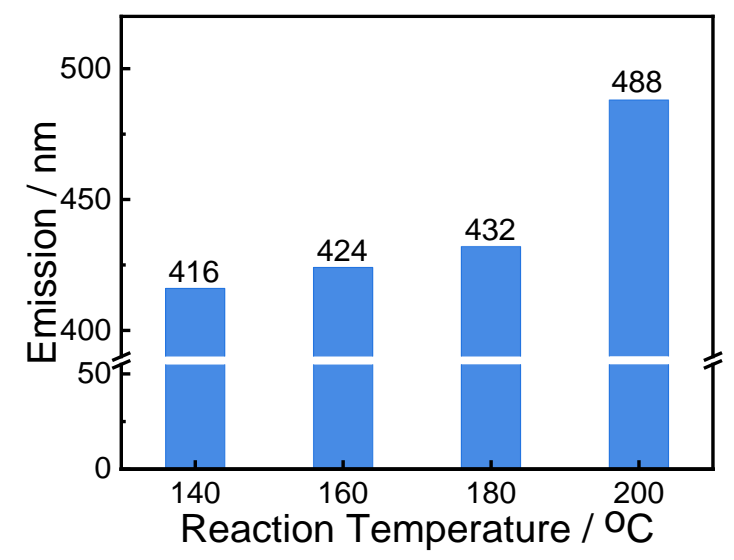

Figure S11. Maximum PL emission peak of the CNQD-CN synthesized at different reaction temperature. Excitation wavelength is $380 \mathrm{~nm}$. 


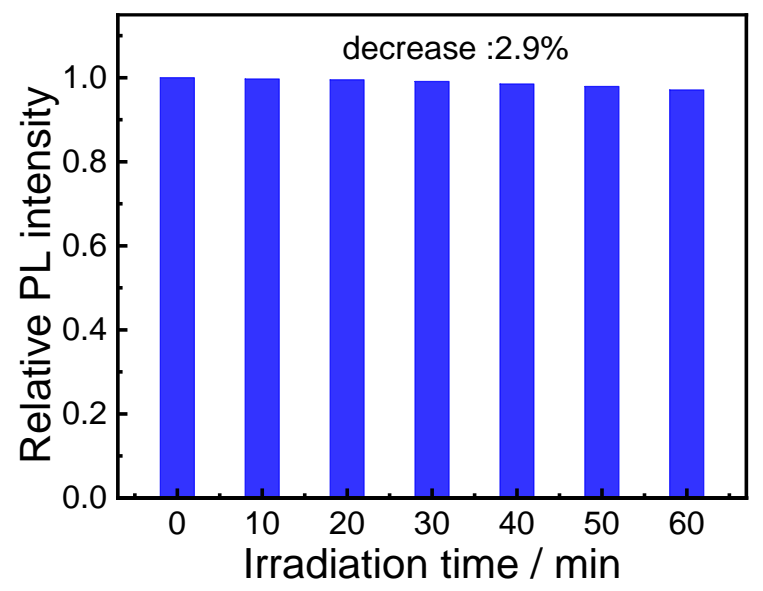

Figure S12. Relative PL intensity change of emission peak (542 nm) of CNQD-CN at an excitation wavelength of $380 \mathrm{~nm}$ with different irradiation time.

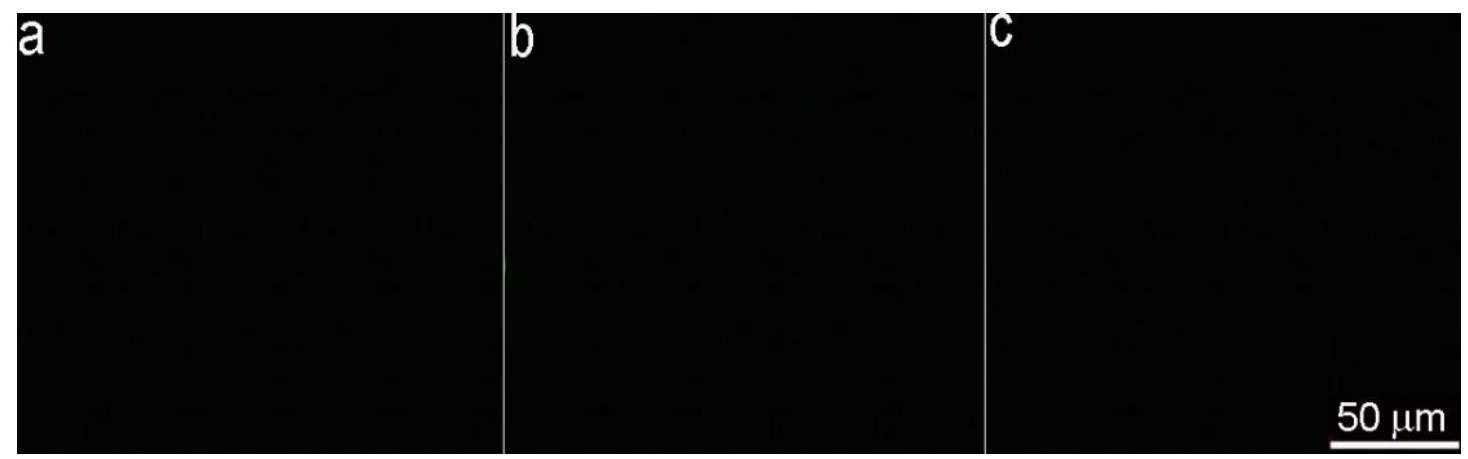

Figure S13. Laser scanning confocal microscopy images of Hela cells incubated with PBS solution for $2 \mathrm{~h}$ under different excitation wavelengths: (a) $405 \mathrm{~nm}$; (b) $488 \mathrm{~nm}$; (c) $546 \mathrm{~nm}$.
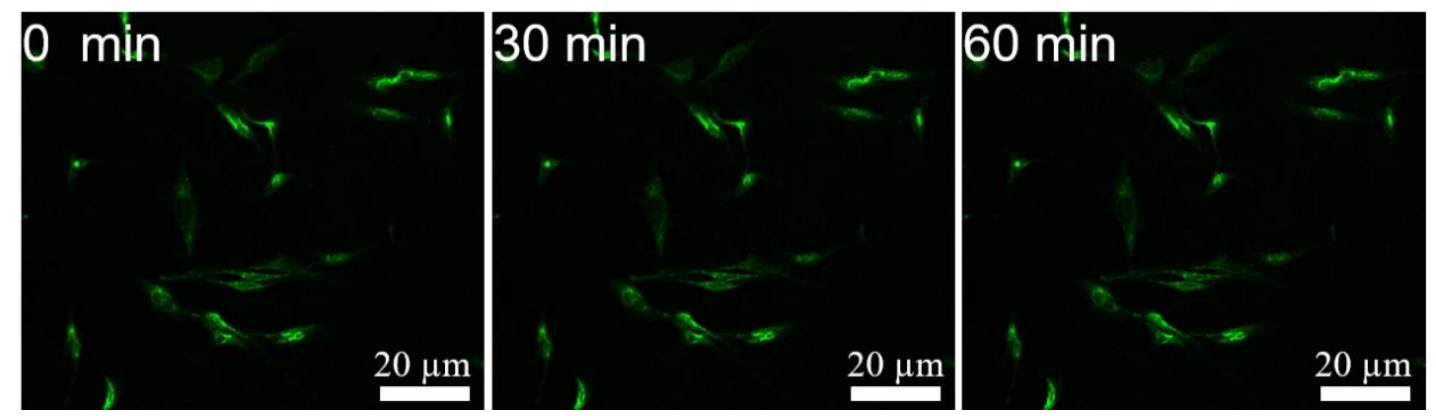

Figure S14. Laser scanning confocal microscopy images of Hela cells incubated with CNQD-CN for $2 \mathrm{~h}$ under different excitation times. (a) $0 \mathrm{~min}$; (b) $30 \mathrm{~min}$; (c) $60 \mathrm{~min}$. Excitation wavelength $=488 \mathrm{~nm}$. 


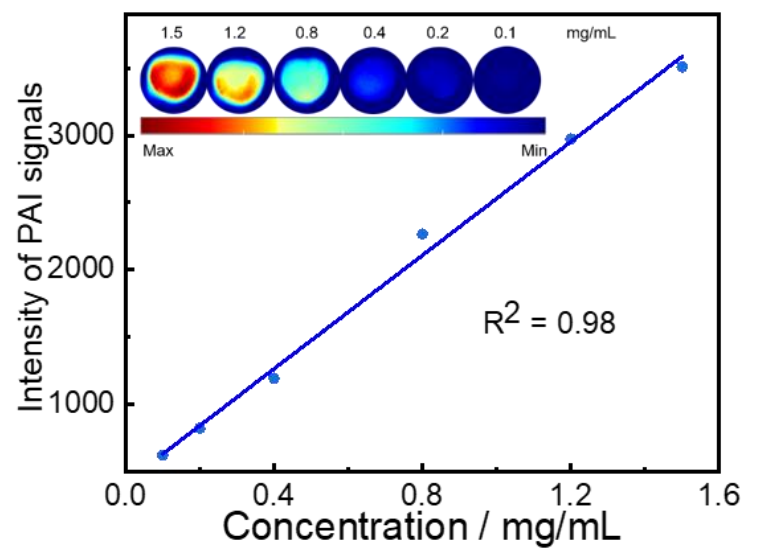

Figure S15. Photoacoustic imaging and linear fitting plot of CNQD-CN aqueous solution at different concentrations using multispectral optical tomography system (MSOT in Vision 256, iThera Medical, Germany) with an excitation wavelength at $808 \mathrm{~nm}$.
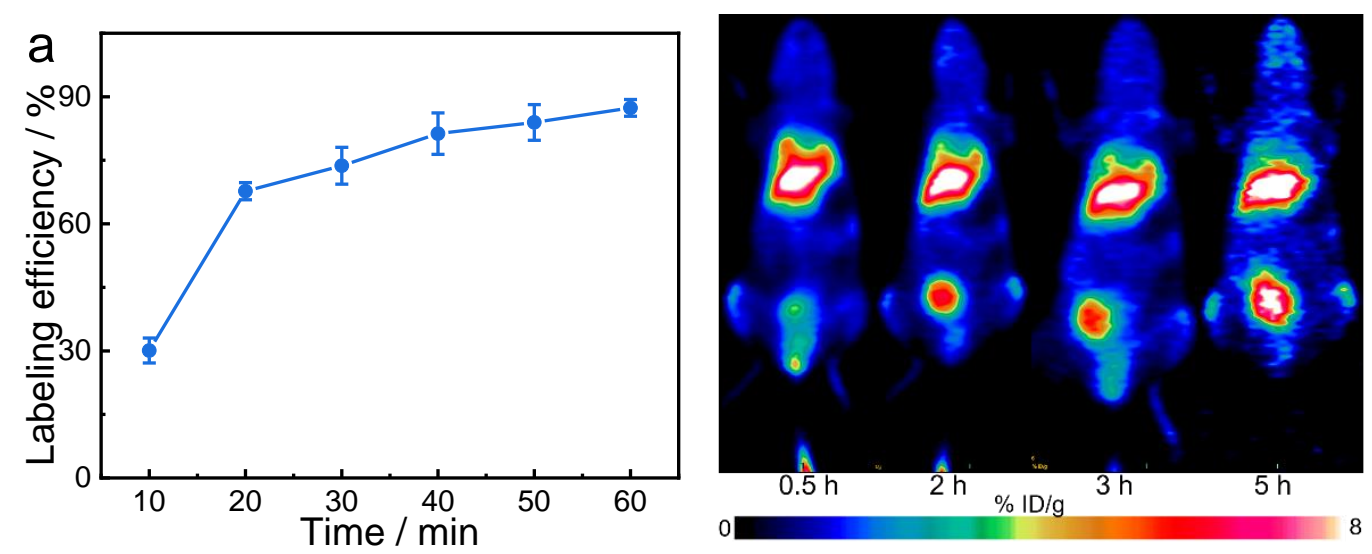

Figure S16. (a) Quantified labeling yield of ${ }^{68} \mathrm{Ga}$ on CNQD-CN at various time points. (b) PET images of HeLa tumor-bearing mice taken at various time points $(0.5,2,3$ and $5 \mathrm{~h}$ ) after i.v. injection of ${ }^{68} \mathrm{Ga}-\mathrm{CNQD}-\mathrm{CN}$. The obvious contrast was observed after $2 \mathrm{~h}$ after injection. 


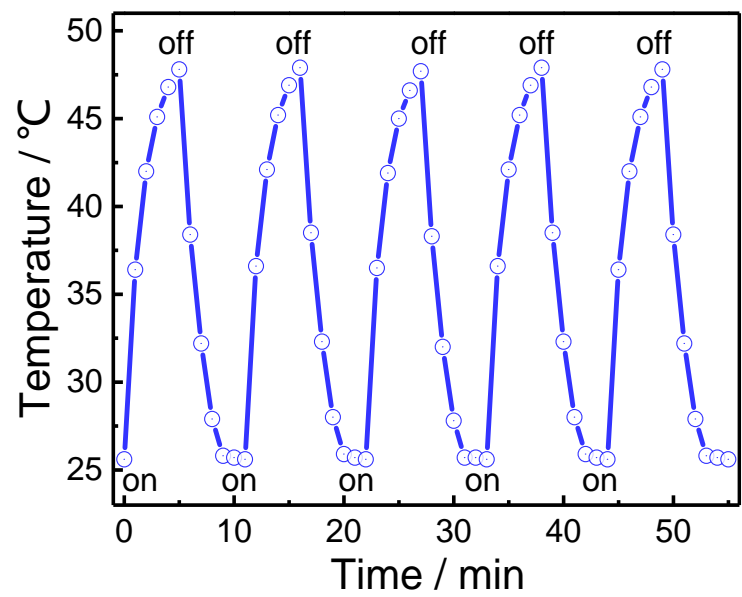

Figure S17. Heating of a suspension of the CNQD-CN in water for five laser on/off cycles.

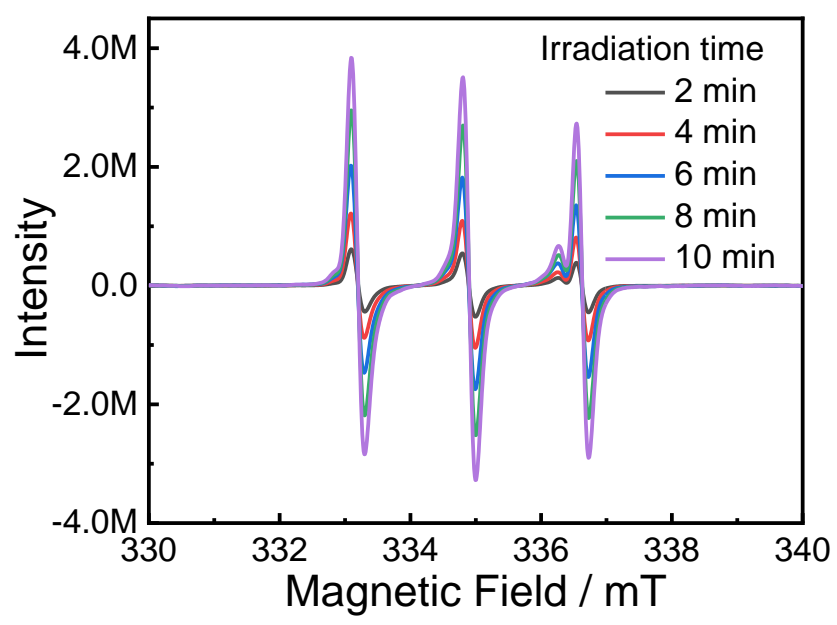

Figure S18. The ESR signals of ${ }^{1} \mathrm{O}_{2}$ obtained upon NIR irradiation of CNQD-CN for different time in the presence of TEMP, respectively.

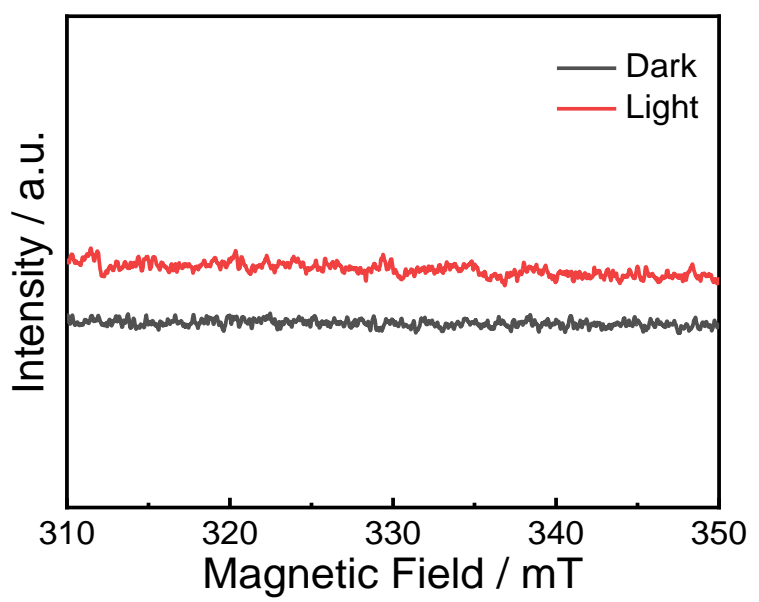

Figure S19. The ESR signals other ROS $(\bullet \mathrm{OH})$ obtained upon irradiation of CNQD-CN for $8 \mathrm{~min}$ in the presence BMPO. 


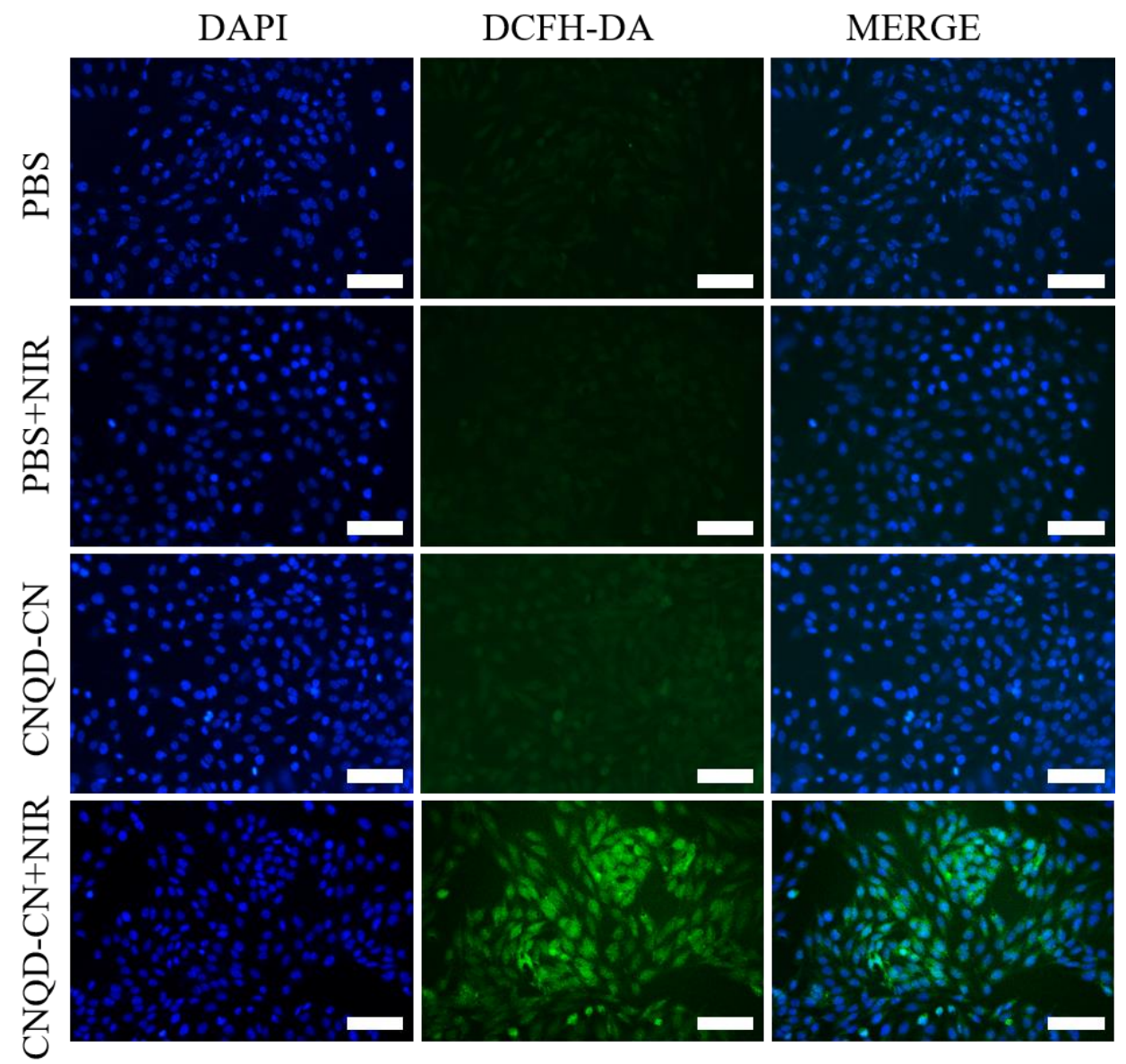

Figure S20. Fluorescence images of Hela cells cultured with/without CNQD-CN (25 $\left.\mu \mathrm{g} \mathrm{mL}^{-1}\right)$ and irradiated with/without an $808 \mathrm{~nm}$ laser $\left(1.0 \mathrm{~W} \mathrm{~cm}^{-2}\right)$. Scale bar is 100 $\mu \mathrm{m}$.

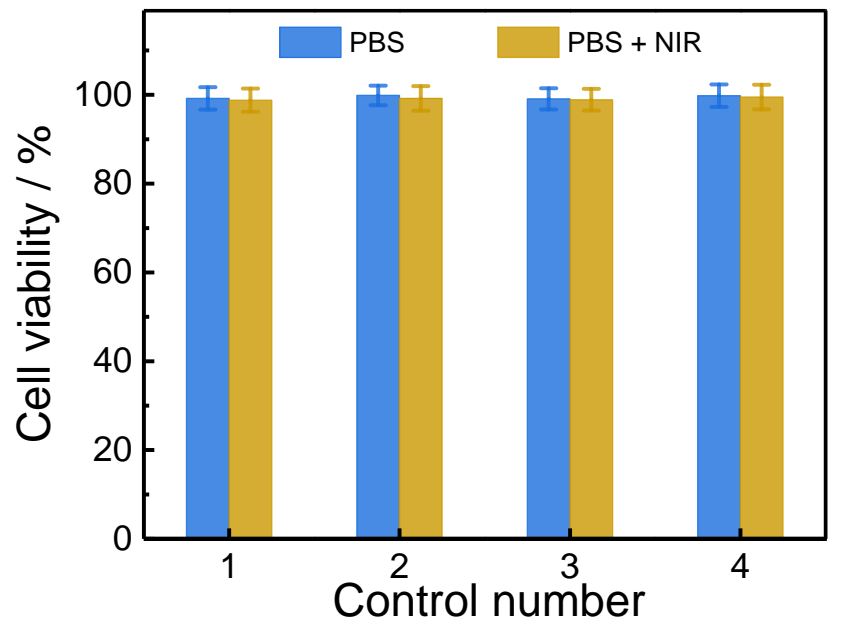

Figure S21. Cell viability in culture media irradiated with/without $1.0 \mathrm{~W} / \mathrm{cm}^{2} \mathrm{NIR}$ irradiation for $5 \mathrm{~min}$. 

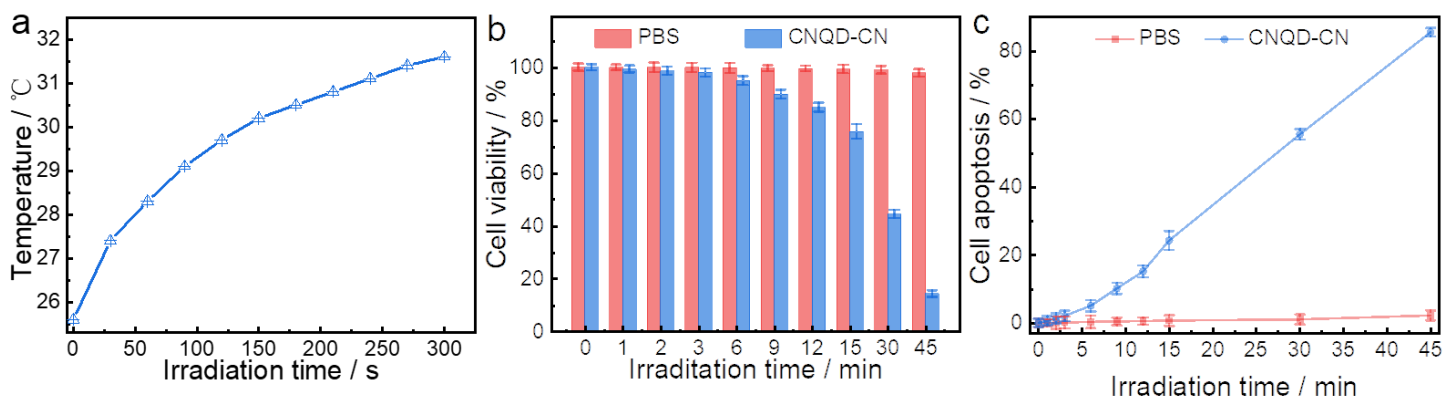

Figure S22. (a) Temperature changes of CNQD-CN dispersions with $6.25 \mu \mathrm{g} \mathrm{mL}^{-1}$ under NIR irradiation ( $\left.808 \mathrm{~nm}, 1.0 \mathrm{~W} / \mathrm{cm}^{2}\right)$ for $300 \mathrm{~s}$. (b and c) Cell viability and cell apoptosis of HeLa cells treated by CNQD-CN with $808 \mathrm{~nm}$ laser $\left(1.0 \mathrm{~W} / \mathrm{cm}^{2}\right)$ at different irradiation time.

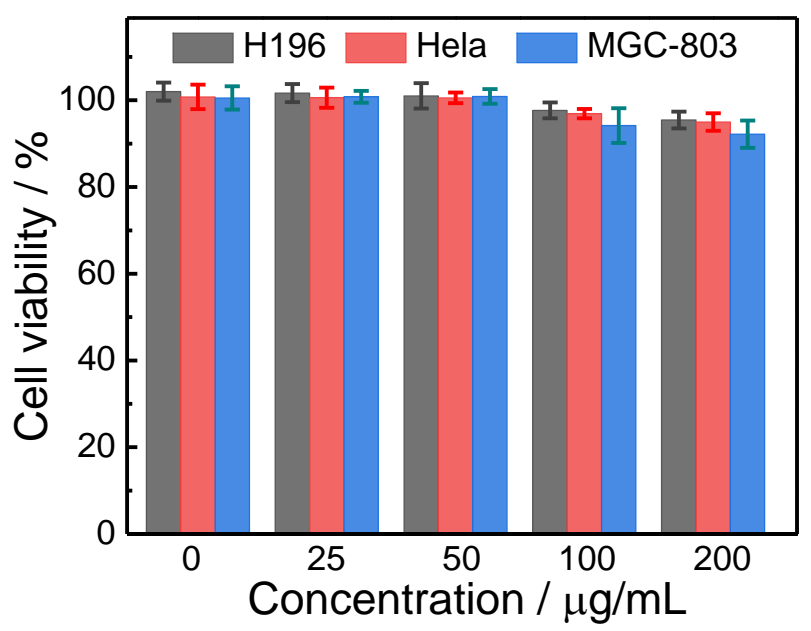

Figure S23. In Vitro cytotoxicity study of CNQD-CN performed by assessing the viability of MGC-803, Hela, and H196 cells $72 \mathrm{~h}$ after treatment with CNQD-CN at different concentrations. 


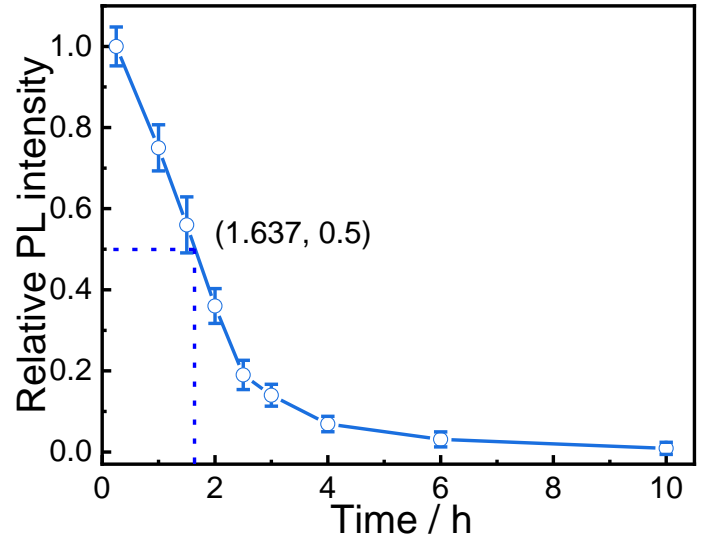

Figure S24. Blood half-life of the CNQD-CN determined by fluorescence measurement.

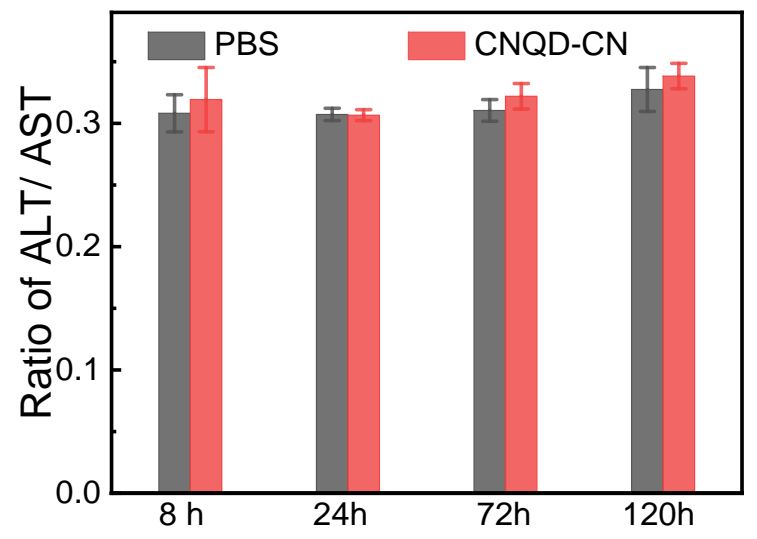

Figure S25. Liver enzyme ratio of ALT to AST from mice treated by PBS (control) and CNQD-CN for $8 \mathrm{~h}, 24 \mathrm{~h}, 72 \mathrm{~h}$ and $120 \mathrm{~h}$. 

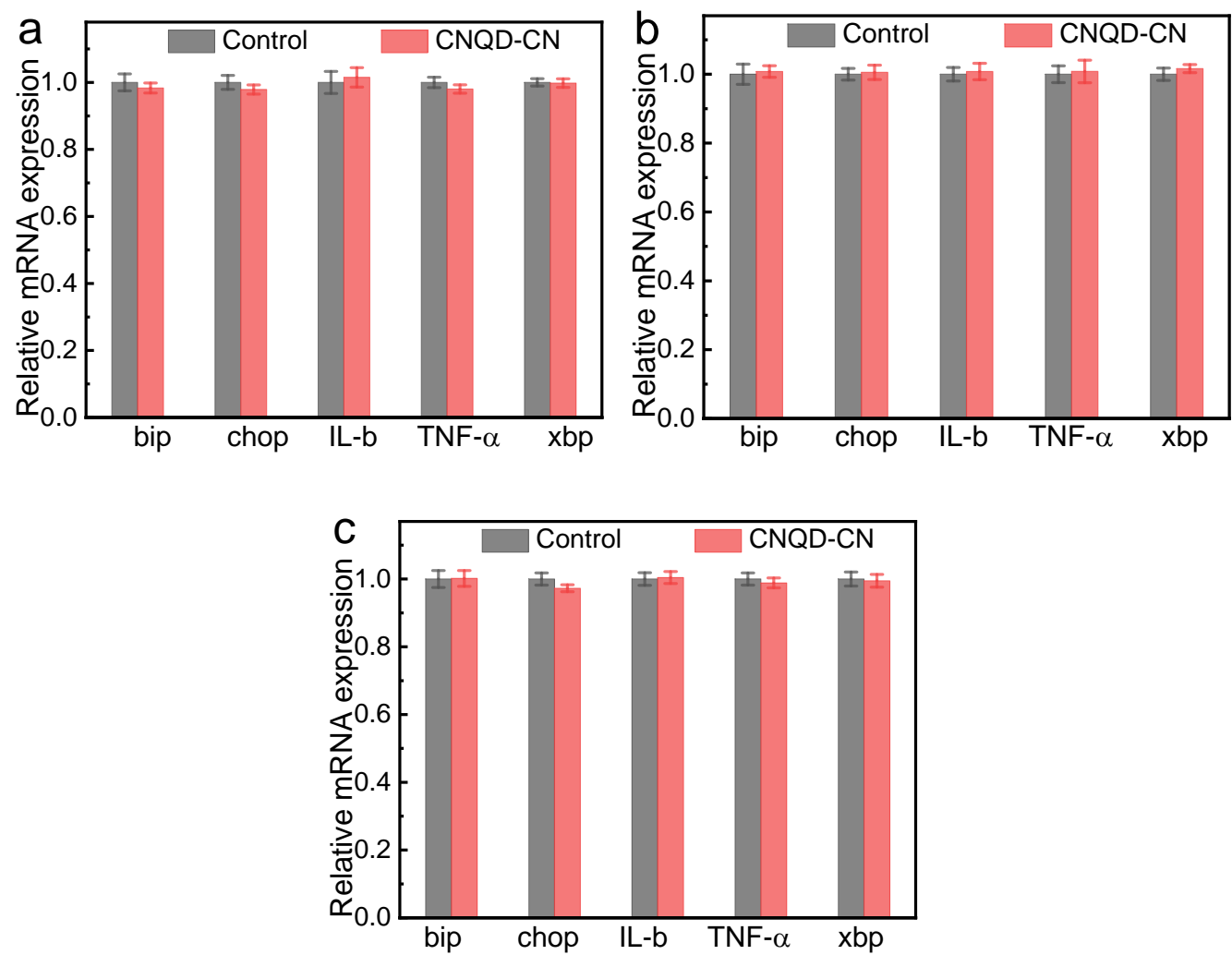

Figure S26. Representative RT-PCR expression level from the tissues of mice treated by PBS (control) and CNQD-CN at post 8 h: (a) kidney, (b) liver, and (c) spleen, respectively.
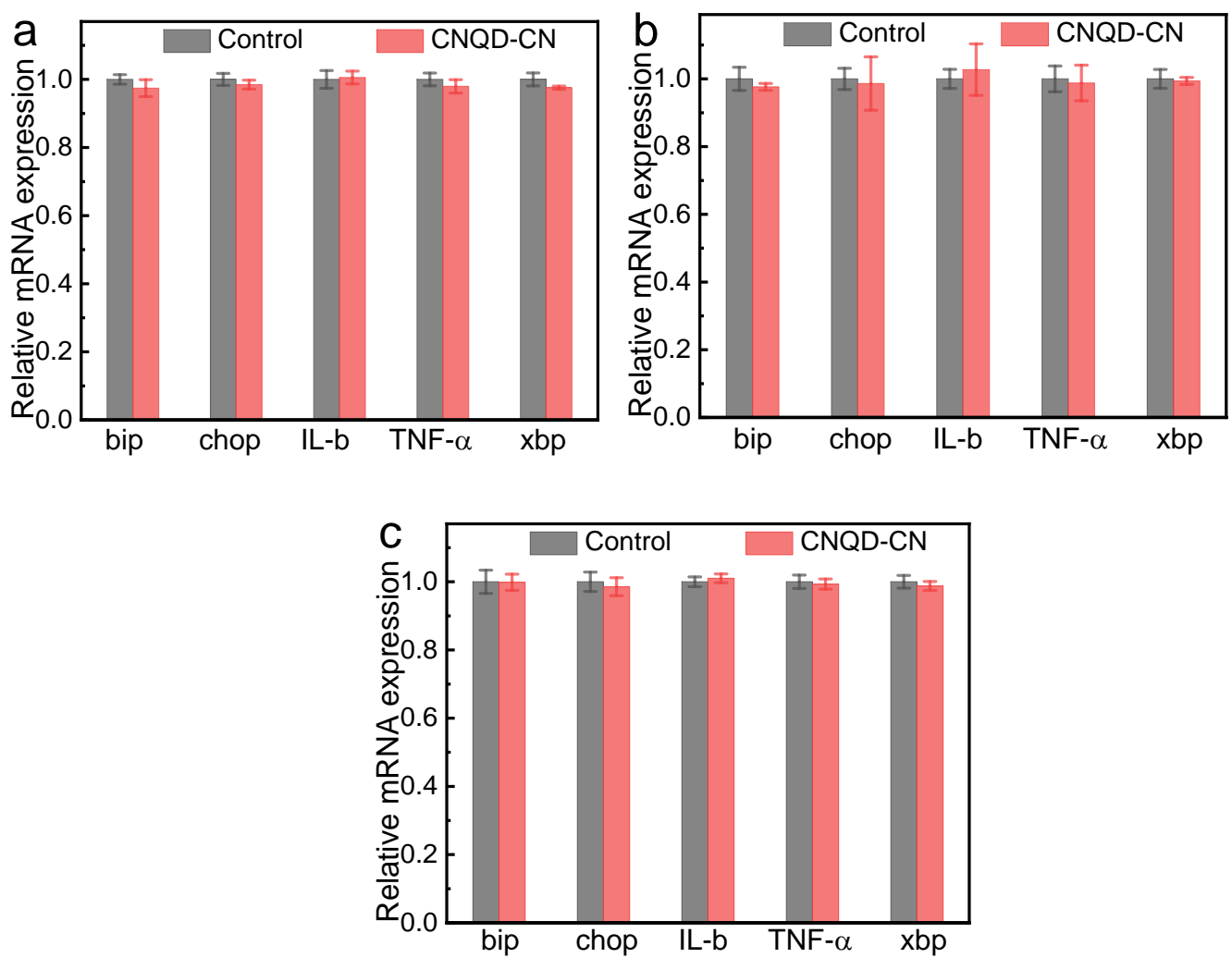
Figure S27. Representative RT-PCR expression level from the tissues of mice treated by PBS (control) and CNQD-CN at post 24 h: (a) kidney, (b) liver, and (c) spleen, respectively.
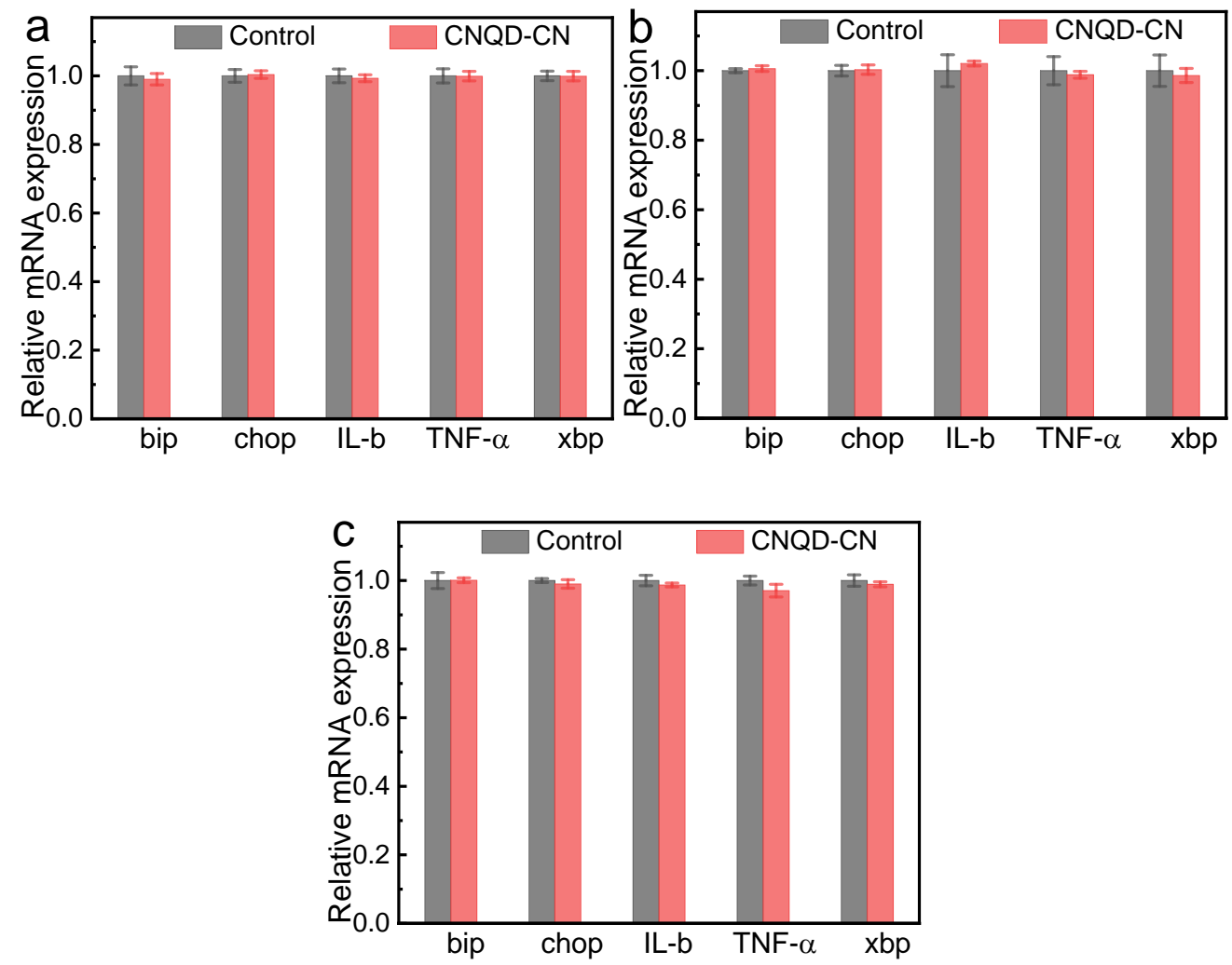

Figure S28. Representative RT-PCR expression level from the tissues of mice treated by PBS (control) and CNQD-CN at post 120 h: (a) kidney, (b) liver, and (c) spleen, respectively.

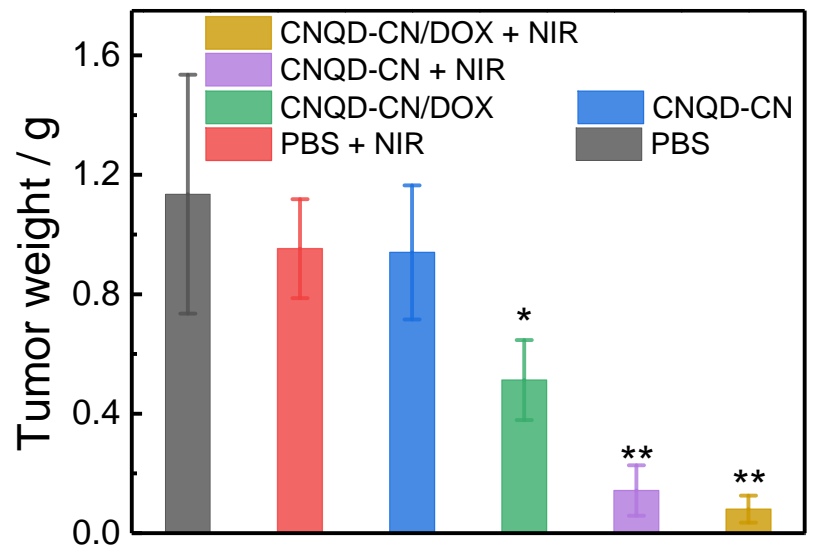

Figure S29. Tumor weight obtained from mice on day 27 after receiving different treatments. 


\section{References:}

1. Kuo, W. S.; Chen, H. H.; Chen, S. Y.; Chang, C. Y.; Chen, P. C.; Hou, Y. I.; Shao, Y. T.; Kao, H. F.; Lilian Hsu, C. L.; Chen, Y. C.; Chen, S. J.; Wu, S. R.; Wang, J. Y., Graphene Quantum Dots with Nitrogen-Doped Content Dependence for Highly Efficient Dual-Modality Photodynamic Antimicrobial Therapy and Bioimaging. Biomaterials 2017, 120, 185-194.

2. Wang, H.; Mu, Q.; Wang, K.; Revia, R. A.; Yen, C.; Gu, X.; Tian, B.; Liu, J.; Zhang, M., Nitrogen and Boron Dual-Doped Graphene Quantum Dots for Near-Infrared Second Window Imaging and Photothermal Therapy. Appl. Mater. Today 2019, 14, 108-117.

3. Wang, H.; Di, J.; Sun, Y.; Fu, J.; Wei, Z.; Matsui, H.; del C. Alonso, A.; Zhou, S., Biocompatible PEG-Chitosan@Carbon Dots Hybrid Nanogels for Two-Photon Fluorescence Imaging, Near-Infrared Light/pH Dual-Responsive Drug Carrier, and Synergistic Therapy. Adv. Funct. Mater. 2015, 25, 5537-5547.

4. Repasky, E. A.; Evans, S. S.; Dewhirst, M. W., Temperature Matters! And Why It Should Matter to Tumor Immunologists. Cancer Immunol. Res. 2013, 1, 210-216. 